\title{
Influence of skeletal class and facial type on nose dimensions in a Brazilian subpopulation: a CBCT study
}

\author{
Amanda Farias GOMES(a) \\ Rocharles Cavalcante \\ FONTENELE(a) \\ Mariana Fabbro ZANON(a) \\ Francisco Carlos GROPPO(b) ID \\ Francisco HAITER NETO(a) \\ Deborah Queiroz FREITAS(a) iD \\ (a) Universidade Estadual de Campinas \\ - Unicamp, Piracicaba Dental School, \\ Department of Oral Diagnosis - Oral \\ Radiology, Piracicaba, SP, Brazil. \\ (b) Universidade Estadual de Campinas \\ - Unicamp, Piracicaba Dental School, \\ Department of Physiological Sciences \\ - Pharmacology, Anesthesiology and \\ Therapeutics, Piracicaba, SP, Brazil.
}

Declaration of Interests: The authors certify that they have no commercial or associative interest that represents a conflict of interest in connection with the manuscript.

Corresponding Author:

Amanda Farias Gomes

E-mail: aamandafg@outlook.com.br

https://doi.org/10.1590/1807-3107bor-2021.vol35.0036

Submitted: Mar 31, 2020

Accepted for publication: October 22, 2020

Last revision: December 9, 2020

\begin{abstract}
Evaluating factors that may influence the nose dimensions and, therefore, contribute to the singularity of a person, is important for obtaining a precise facial reconstruction (FR) in forensic anthropology. Thus, the aim in this study was to evaluate the influence of sex, skeletal class, and facial type on nose dimensions of Brazilian individuals using cone-beam computed tomography (CBCT) scans. CBCT images of 54 males and 67 females were classified according to skeletal class (class I, II, or III) and facial type (brachycephalic, dolichocephalic and mesocephalic). Linear and angular measurements of the nose were performed using the CS 3D Imaging software. The measurements obtained for males and females were compared by one-way ANOVA. Two-way ANOVA was used to compare the measurements in the different skeletal classes and facial types. Significance level was set at $5 \%$. All linear measurements were greater in males $(p<0.05)$; the angular measurements, however, did not differ between sexes ( $p>0.05)$. Linear and angular measurements of class III males were significantly different from the other classes; the same was observed for the angular measurements in females $(\mathrm{p}<0.05)$. Regarding facial types, some linear measurements were significantly greater in dolichocephalics males $(p<0.05)$. For females, there was no influence of facial type $(p>0.05)$, except for the nasal convexity angle. Nose dimensions differed in specific points among different skeletal classes and facial types, and there was sexual dimorphism in all linear nose dimensions. This information may be applicable for nose reconstructions, allowing more reliable FR.
\end{abstract}

Keywords: Forensic Anthropology; Nose; Forensic Dentistry; Cone-Beam Computed Tomography.

\section{Introduction}

Human postmortem identification represents a challenge for anthropology and forensic medicine. The primary identification techniques are based on comparative analysis between antemortem and postmortem registries, such as fingerprinting, DNA analysis, and dental comparative records. ${ }^{1,2,3}$ However, identification by primary methods is impracticable when there is insufficient information for comparison or it is not conclusive. In these situations, facial reconstruction (FR) can be used to assist in the recognition of severely damaged human remains. ${ }^{4,5}$ It is not a method of 
identification, but a tool used for recognition. The publication of the reconstructed face is expected to facilitate familial recognition, generating a restricted list of suspects, from which the subject can be identified through the primary identification methods. ${ }^{6}$

FR is an auxiliary technique in human identification that consists in recreating the face of an individual by studying the relationship between the skull bones and the thickness of the soft tissues that cover the bone at specific craniometric points. ${ }^{4}$ Previous studies have suggested that the anthropomorphometric features of the face are influenced by several factors such as sex, ethnicity, age, and skeletal classes. ${ }^{7,9,9,10}$ Thus, substantial knowledge about the biologic profile, craniofacial musculature, and skeletal anatomy is of great importance for human recognition through the FR technique, since the relationship between these factors contributes to the facial singularity of each individual. ${ }^{1,3,11}$

Nasal morphology is an important feature for a reliable FR as the nose is the most central and prominent structure of the human face.,12 The position, shape, size and symmetry of the nose are determining elements for facial expression, and its dimensions significantly affect the facial soft tissue form and the degree of profile convexity. ${ }^{9}$ Unfortunately, the nose shape is lost during cadaveric decomposition, since its constitution is mainly cartilage and soft tissue, which may decrease FR accuracy. ${ }^{9}$ Therefore, establishing dimensional parameters that facilitate nose reconstruction is important to obtain a precise FR.

The nose morphology is often manually reconstructed from the shape and size of the nasal aperture, using as reference the pronasal, subnasal, and alar points. ${ }^{10}$ However, the utilization of cadaver samples may cause distortion of the soft tissue data as desiccation occurs within few hours after death. ${ }^{10}$ In an attempt to overcome the limitations of postmortem desiccation, some authors developed methods for estimating anthropometric characteristics in living populations using digital imaging, such as cephalometric radiography, computed tomography, and magnetic resonance. ${ }^{10,13,14}$

Recent studies recommended the use of cone beam computed tomography (CBCT) to assess the relationship between soft tissues and skeletal components of the nose to predict nasal morphology. ${ }^{10,15}$ This technique has advantages compared to cephalometric radiography, such as the three-dimensional evaluation of the scanned structures. When compared with multidetector computed tomography (MDCT), CBCT presents advantages such as lower cost, relatively lower dose of radiation, and higher spatial resolution. ${ }^{16}$ Moreover, the acquisition of CBCT images can be performed with the patient in the sitting or upright position, which may decrease the interference of gravity in the soft tissues thickness. ${ }^{17}$

Previous studies have suggested that nose morphology is strongly related to age and sex in several populations. ${ }^{18,19,20}$ Additionally, some have demonstrated the existence of a relationship between the thickness of the craniofacial soft tissues and different skeletal classes and/or facial profiles. ${ }^{5,14,21,22}$ However, according to the consulted literature, there is a lack of information about the relationship between these factors and nasal dimensions in the Brazilian population. ${ }^{13}$ Therefore, the aim in this study was to evaluate the influence of sex, skeletal class (class I, II and III), and facial type (brachycephalic, mesocephalic and dolichocephalic) on the nasal dimensions of Brazilian individuals, using CBCT images.

\section{Methodology}

This retrospective cross-sectional study was approved by the local Research Ethics Committee without restrictions (protocol: CAAE 70501317.3.0000.5418) and was conducted according to the Helsinki Declaration.

\section{Sample selection}

CBCT scans of individuals aged 21 years or older, with satisfactory image quality (i.e., adequate image density, contrast, and definition) for visualization and delimitation of bone structures and facial profile were used as inclusion criteria. The exclusion criteria were absence of upper incisors, presence of dental implant in the anterior region, past or current orthodontic treatment and/or orthognathic surgery, maxillofacial trauma, pathologies in the head and neck regions, craniofacial syndromes, and image artefacts impairing the visualization of the landmarks to be studied. 
The CBCT images were obtained from the database of a local radiology clinic in the state of São Paulo, and all patients were from the southeast region of Brazil. Images were acquired previously to the present study for orthodontic or orthognathic surgery planning, using an i-CAT Next Generation (Imaging Science, Hatfield, USA) unit with the following parameters: $120 \mathrm{kVp}, 5 \mathrm{~mA}, 23 \times 17 \mathrm{~cm}$ field of view, $0.4 \mathrm{~mm}^{3}$ voxel size, $40 \mathrm{~s}$ acquisition time, and $62 \mathrm{~s}$ reconstruction time.

\section{Sample classification}

Skeletal class (classes I, II, and III) and facial type (brachycephalic, dolichocephalic, and mesocephalic) were determined for each individual using multiplanar reconstructions (lateral cephalometric) derived from the CBCT scans, using the NemoCeph software (Nemotec, Madrid, Spain).

The skeletal class was determined by means of the SNA, SNB, and ANB angles from the Steiner's cephalometric analysis ${ }^{23}$ and confirmed by the Jarabak and Fizzell measurement. ${ }^{24}$ The facial type was determined through the VERT index as calculated in the Ricketts' cephalometric analysis. ${ }^{25}$

\section{Measurements}

Linear (Table 1 and Figure 1A-B) and angular (Table 2 and Figure 2A-D) measurements were performed on a computer display (Dell 14-inch liquid-crystal display monitor with 1,366 x 768 resolution; Dell, Round Rock, TX, USA), using the CS 3D Imaging software 3.5.18 (Carestream Health, Rochester, NY, USA). Two oral radiologists discussed the landmarks' location in each CBCT scan and, after they reached consensus, one of them performed the measurements. Before the measurement, each scan was spatially oriented so that the anterior and posterior nasal spines were visualized in the same axial and sagittal reconstructions. The analyses were conducted in a silent and dimmed-light room, and the examiners were allowed to adjust brightness, contrast, and zoom as appropriate. The scans were evaluated in maximum intensity of pixel (MIP), with $10.8 \mathrm{~mm}$ thickness for sagittal and $16.4 \mathrm{~mm}$ thickness for axial reconstructions.

Thirty days after completion of the evaluations, $30 \%$ of the sample was re-evaluated under the same conditions to test the intra-examiner agreement.

Table 1. Linear measurements' protocol.

\begin{tabular}{|c|c|c|c|}
\hline Measurement & Anatomical description & Reconstruction & Figure \\
\hline Length 1 & Distance from nasion to pronasale & Sagittal & $1 A$ \\
\hline Height & Distance from nasion to subnasale & Sagittal & $1 A$ \\
\hline Depth & Distance from subnasale to pronasale & Sagittal & $1 \mathrm{~A}$ \\
\hline Width 1 & Distance between right and left alar points & Axial & $1 \mathrm{~B}$ \\
\hline Width 2 & Distance between right and left nasal wings & Axial & $1 \mathrm{~B}$ \\
\hline Length 2 & Distance from the center of the alar base to the pronasale & Axial & $1 \mathrm{~B}$ \\
\hline
\end{tabular}


Figure 1. CBCT cropped multiplanar reconstructions illustrating the linear measurements of the nose. A - 1. Length $1 ; 2$. Height; 3. Depth. B -4 . Width 1; 5. Width 2; 6. Length 2. 
Table 2. Angular measurements' protocol.

\begin{tabular}{|c|c|c|c|}
\hline Measurement & Description & Reconstruction & Figure \\
\hline Naso-frontal angle (NFA) & $\begin{array}{l}\text { Angle with the vertex at the nasion, formed by a tangent line to the frontal bone } \\
\text { in the frontal bone prominence and a tangent line to the nose }\end{array}$ & Sagittal & $2 \mathrm{~A}$ \\
\hline Naso-labial angle (NLA) & $\begin{array}{l}\text { Angle with the vertex at the subnasal point, formed by a tangent line to the nasal } \\
\qquad \text { tip and a tangent line to the upper lip }\end{array}$ & Sagittal & $2 \mathrm{~A}$ \\
\hline Nasal convexity angle (NCA) & Angle with the vertex at the nasion, formed by the lines of length 1 and height & Sagittal & 2B \\
\hline Nasal projection angle (NPA) & Angle with the vertex at the pronasal, formed by the lines of length 1 and depth & Sagittal & $2 \mathrm{C}$ \\
\hline Interalar angle (IAA) & $\begin{array}{c}\text { Angle with the vertex at the pronasal, formed by the tangent lines to the right and } \\
\text { left alar points }\end{array}$ & Axial & $2 \mathrm{D}$ \\
\hline
\end{tabular}


Figure 2. CBCT cropped multiplanar reconstructions illustrating the angular measurements of the nose. $A$, Sagittal reconstruction - 1. Naso-frontal angle (NFA); 2 - Naso-labial angle (NLA). B, Sagittal reconstruction - 3. Nasal convexity angle (NCA). C, Sagittal reconstruction - 4. Nasal projection angle (NPA). D, Axial reconstruction - 5. Interalar angle (IAA).

\section{Statistical analysis}

Intra-examiner agreement regarding the linear and angular measurements was assessed by the intra-class correlation coefficient (ICC) $(<0.40$, poor agreement; 0.40 to 0.59 , fair; 0.60 to 0.74 , good; 0.75 to 1.00, excellent). ${ }^{26}$ The measurements obtained for males and females were compared by one-way ANOVA. The two-way ANOVA with post-hoc Tukey test was employed to compare the measurements in the different skeletal classes and facial types. Since ANOVA showed differences between sexes, the influence of skeletal classes and facial types was tested independently for each sex. Such influence was tested together; however, the results are shown in two tables to better show the differences. Power analysis was performed with the values obtained in the present study. Considering the differences among the skeletal classes and the facial types for all measurements employed in the study and the variability of these measurements (mean, SD), a statistical power of $80 \%$ was found for all of them. The null hypothesis considered that sex, skeletal class, and facial type do not influence the nasal dimensions. All data were statistically analyzed using the SPSS v. 24.0 (IBM Corp, Armonk, NY, USA), with a significance level of $5 \%(p<0.05)$.

\section{Results}

One hundred and twenty-one CBCT scans of 54 males and 67 females, aged 21 to 40 years (mean age 28 years old) composed the study sample.

The ICC showed an excellent intra-examiner agreement $(0.997, \mathrm{p}<0.0001)$ when all measurements were considered together. The same level of agreement was observed when the measurements were independently evaluated: length 1 (0.957), height (0.955), depth (0.864), width 1 (0.951), width 2 (0.939), length 2 (0.952), NFA (0.977), NLA (0.990), NCA (0.893), NPA (0.972), and IAA (0.946). 
The sample distribution according to skeletal class and facial type is shown in Table 3, in which similar distribution among the studied factors was observed.

All linear measurements were greater in males $(p<0.05)$; the angular measurements, however, did not differ between sexes ( $p>0.05)$. (Table 4).

Regarding the influence of the skeletal class on nasal dimensions, class III males had greater values of nasal height $(p=0.046)$, NFA ( $p=0.046)$, and NPA $(p=0.038)$ than class II males. Greater values of width 2 in class III than in class I males $(p=0.042)$, and greater NLA in class I than in class III males $(p=0.026)$ were also found. In the female group, the skeletal class influenced only angular measurements. Class III females presented greater values of NCA than class I and class II females $(p=0.002)$. The NLA values, on the other hand, were greater in class I and class II than in class III females $(p=0.007)$. Class I also presented greater values of NPA than class III females $(p=0.023)($ Table 5).
In relation to the facial type, dolichocephalic males presented greater values of length $1(p=0.013)$ and height $(p=0.014)$ than the brachycephalic ones. Dolichocephalic males also showed greater values of length 2 than mesocephalics ( $p=0.019$ ). The NCA dimension was greater in brachycephalics than in mesocephalics $(p=0.032)$. In females, only the NCA dimension differed among the facial types, with brachycephalics presenting greater values than dolichocephalics $(p=0.038)$ (Table 6).

\section{Discussion}

Reconstruction of the nasal region is one of the most critical stages of the FR due to the complexity of this anatomical structure and subjectivity of the method., ${ }^{9}$ Therefore, studies about the influence of different factors such as sex, age, and ethnicity on nose dimensions have been performed to provide data for a more reliable reconstruction. ${ }^{1,9,18,19,27}$ Although

Table 3. Sample distribution according to skeletal class and facial type.

\begin{tabular}{lccc}
\hline Variable & Male $(\mathrm{n}=54)$ & Female $(\mathrm{n}=67)$ & Total $(\mathrm{n}=121)$ \\
\hline Class I & $21(38.9 \%)$ & $29(43.3 \%)$ & $50(41.3 \%)$ \\
Class II & $12(22.2 \%)$ & $21(31.3 \%)$ & $33(27.3 \%)$ \\
Class III & $21(38.9 \%)$ & $17(25.4 \%)$ & $38(31.4 \%)$ \\
Brachycephalic & $25(46.3 \%)$ & $28(41.8 \%)$ & $53(43.8 \%)$ \\
Dolichocephalic & $17(31.5 \%)$ & $18(26.9 \%)$ & $35(28.9 \%)$ \\
Mesocephalic & $12(22.2 \%)$ & $21(31.3 \%)$ & $33(27.3 \%)$ \\
\hline
\end{tabular}

Table 4. Mean ( \pm standard deviation) of the linear (millimeters) and angular (degrees) measurements of the nose according to sex.

\begin{tabular}{lccc}
\hline \multirow{2}{*}{ Measurement } & Male $(\mathrm{n}=54)$ & Female $(\mathrm{n}=67)$ & $\mathrm{p}$-value \\
\cline { 2 - 3 } Length 1 & (Mean \pm SD) & $($ Mean \pm SD) & 0.000 \\
Height & $51.78( \pm 3.76)$ & $48.71( \pm 2.81)$ & 0.000 \\
Depth & $57.84( \pm 3.70)$ & $54.02( \pm 2.86)$ & 0.000 \\
Width 1 & $18.12( \pm 2.11)$ & $16.49( \pm 1.81)$ & 0.000 \\
Width 2 & $37.92( \pm 3.39)$ & $33.74( \pm 2.62)$ & 0.000 \\
Length 2 & $34.94( \pm 4.29)$ & $31.50( \pm 3.54)$ & 0.000 \\
NFA & $28.44( \pm 2.57)$ & $25.60( \pm 1.80)$ & 0.963 \\
NCA & $141.85( \pm 9.58)$ & $141.92( \pm 7.60)$ & $17.41( \pm 2.23)$ \\
NPA & $17.85( \pm 2.54)$ & $99.74( \pm 5.77)$ & 0.320 \\
NLA & $99.85( \pm 6.90)$ & $105.02( \pm 10.89)$ & 0.927 \\
IAA & $105.37( \pm 15.59)$ & $81.37( \pm 6.24)$ & 0.888 \\
\hline
\end{tabular}


Table 5. Mean ( \pm standard deviation) of the linear (millimeters) and angular (degrees) measurements in the nose, according to skeletal class in males and females.

\begin{tabular}{|c|c|c|c|c|c|c|c|c|}
\hline \multirow{4}{*}{ Measurement } & \multicolumn{4}{|c|}{ Males $(n=54)$} & \multicolumn{4}{|c|}{ Females $(n=67)$} \\
\hline & \multicolumn{3}{|c|}{ Skeletal Class } & \multirow{3}{*}{$\mathrm{p}$-value } & \multicolumn{3}{|c|}{ Skeletal Class } & \multirow{3}{*}{$\mathrm{p}$-value } \\
\hline & Class I & Class II & Class III & & Class I & Class II & Class III & \\
\hline & $\mathrm{n}=21$ & $\mathrm{n}=12$ & $\mathrm{n}=21$ & & $\mathrm{n}=29$ & $\mathrm{n}=21$ & $\mathrm{n}=17$ & \\
\hline Length 1 & $51.28( \pm 4.25) \mathrm{A}$ & $53.00( \pm 3.47) \mathrm{A}$ & $51.58( \pm 3.40) \mathrm{A}$ & 0.438 & $48.35( \pm 2.41) \mathrm{A}$ & $49.74( \pm 3.34) \mathrm{A}$ & $48.04( \pm 2.56) \mathrm{A}$ & 0.119 \\
\hline Height & $57.51( \pm 3.90) A B$ & $59.97( \pm 2.68) \mathrm{A}$ & $56.94( \pm 3.66) \mathrm{B}$ & 0.046 & $54.06( \pm 2.53) \mathrm{A}$ & $54.63( \pm 3.49) \mathrm{A}$ & $53.22( \pm 2.45) \mathrm{A}$ & 0.323 \\
\hline Depth & $18.30( \pm 1.99) \mathrm{A}$ & $18.29( \pm 2.78) \mathrm{A}$ & $17.83( \pm 1.85) \mathrm{A}$ & 0.735 & $16.26( \pm 1.87) \mathrm{A}$ & $16.14( \pm 1.51) \mathrm{A}$ & $17.32( \pm 1.87) \mathrm{A}$ & 0.088 \\
\hline Width 1 & $37.00( \pm 3.94) \mathrm{A}$ & $37.75( \pm 3.18) \mathrm{A}$ & $38.93( \pm 2.71) \mathrm{A}$ & 0.181 & $33.78( \pm 2.34) \mathrm{A}$ & $33.20( \pm 3.32) \mathrm{A}$ & $34.34( \pm 2.03) \mathrm{A}$ & 0.415 \\
\hline Width 2 & $33.32( \pm 4.76)$ B & $35.03( \pm 4.07) A B$ & 36.50 (3.43) A & 0.042 & $31.35( \pm 3.32) \mathrm{A}$ & $31.14( \pm 4.11) \mathrm{A}$ & $32.19( \pm 3.23) \mathrm{A}$ & 0.641 \\
\hline Length 2 & $28.23( \pm 2.34) \mathrm{A}$ & $28.33( \pm 3.36) \mathrm{A}$ & $28.71( \pm 2.38) \mathrm{A}$ & 0.826 & $25.19( \pm 1.78) \mathrm{A}$ & $26.12( \pm 1.74) \mathrm{A}$ & $25.67( \pm 1.93) \mathrm{A}$ & 0.196 \\
\hline NFA & $143.5( \pm 7.80) A B$ & $145.6( \pm 9.14) \mathrm{A}$ & $137.9( \pm 10.4) \mathrm{B}$ & 0.046 & $143.5( \pm 5.84) \mathrm{A}$ & $140.6( \pm 9.89) \mathrm{A}$ & $140.7( \pm 6.94) \mathrm{A}$ & 0.315 \\
\hline NCA & $18.14( \pm 2.59) \mathrm{A}$ & $17.16( \pm 2.97) \mathrm{A}$ & $17.95( \pm 1.26) \mathrm{A}$ & 0.564 & $16.93( \pm 1.88) B$ & $16.80( \pm 1.74) B$ & $19.00( \pm 2.64) \mathrm{A}$ & 0.002 \\
\hline NPA & $100.2( \pm 6.84) A B$ & $103.5( \pm 6.50) \mathrm{A}$ & 97.33 (6.41) B & 0.038 & $101.8( \pm 5.36) \mathrm{A}$ & 98.7 (5.56) $A B$ & $97.41(5.78) \mathrm{B}$ & 0.023 \\
\hline NLA & $110.6( \pm 13.08) \mathrm{A}$ & $108.3( \pm 14.52) A B$ & 98.38 (16.47) A & 0.026 & $107.8( \pm 9.41) \mathrm{A}$ & 106.8 (9.42) A & 98.0 (12.38) B & 0.007 \\
\hline IAA & $81.09( \pm 7.48) \mathrm{A}$ & $81.58( \pm 9.36) \mathrm{A}$ & $81.90( \pm 5.94) \mathrm{A}$ & 0.939 & $82.93( \pm 5.64) \mathrm{A}$ & $79.19( \pm 7.08) \mathrm{A}$ & $81.41( \pm 5.63) \mathrm{A}$ & 0.112 \\
\hline
\end{tabular}

some researchers suggested that the facial form and dimensions are not exclusively defined by the underlying skeletal tissues, the relationship between skeletal and soft tissue parameters could provide valuable information about the strength of correlation and accuracy of the prediction process. ${ }^{9}$ Bearing this in mind and because recent studies have reported a relationship between facial skeletal pattern and soft tissue thickness, ${ }^{5,14,28}$ we considered that it would be of interest to test if skeletal class and facial type have an influence on nose dimensions. Our results indicated significant differences between skeletal classes and facial types for some of the nose dimensions evaluated.

The study of sexual dimorphism from linear and angular measurements of nasal structures was the aim of previous studies conducted with different ethnic groups. ${ }^{1,913,27}$ The present study, from the analysis of 6 linear and 5 angular measurements, found that males had significantly greater values than females for all linear measurements. In agreement with our results, previous studies in different populations, such as Italian, ${ }^{27}$ Chinese, ${ }^{28}$ Scottish and Indonesian, ${ }^{1}$ reported greater values of nose dimensions in men. Therefore, we believe that these differences between sexes should be taken into consideration in FR and also in future studies about sexual dimorphism. The angle measurements, on the other hand, did not show differences between sexes in our sample.
He et al., ${ }^{12}$ who used photometry to evaluate the morphological characteristics of the nose in the Chinese population, observed sexual dimorphism in angular nasal dimensions, with females presenting grater values than males. The difference between this and our results may be explained by ethnic variations and the method employed for evaluation.

Utsuno et al. ${ }^{14}$ emphasized the importance of studying nasal morphology in different skeletal classes, owing to the scarcity of researches on this matter and the importance of the nose for facial physiognomy. Through our results, we confirmed the importance raised by these authors, as nose dimensions differed in specific points in different skeletal classes. The results showed a similarity in nose profile of class I and class II individuals, which differed in some points from the class III subjects. According to the consulted literature, only one study evaluated nose dimensions in subjects of different skeletal classes. ${ }^{28}$ Nevertheless, our results cannot be compared to theirs, since they compared nose dimensions between sexes within the same skeletal class and did not assess differences among skeletal classes.

Regarding the influence of facial types on nose dimensions, we believe that the greater NCA in brachycephalics may be attributed to the characteristic short nose, with rounded and upward tip that these individuals present. The dolichocephalic individuals, 


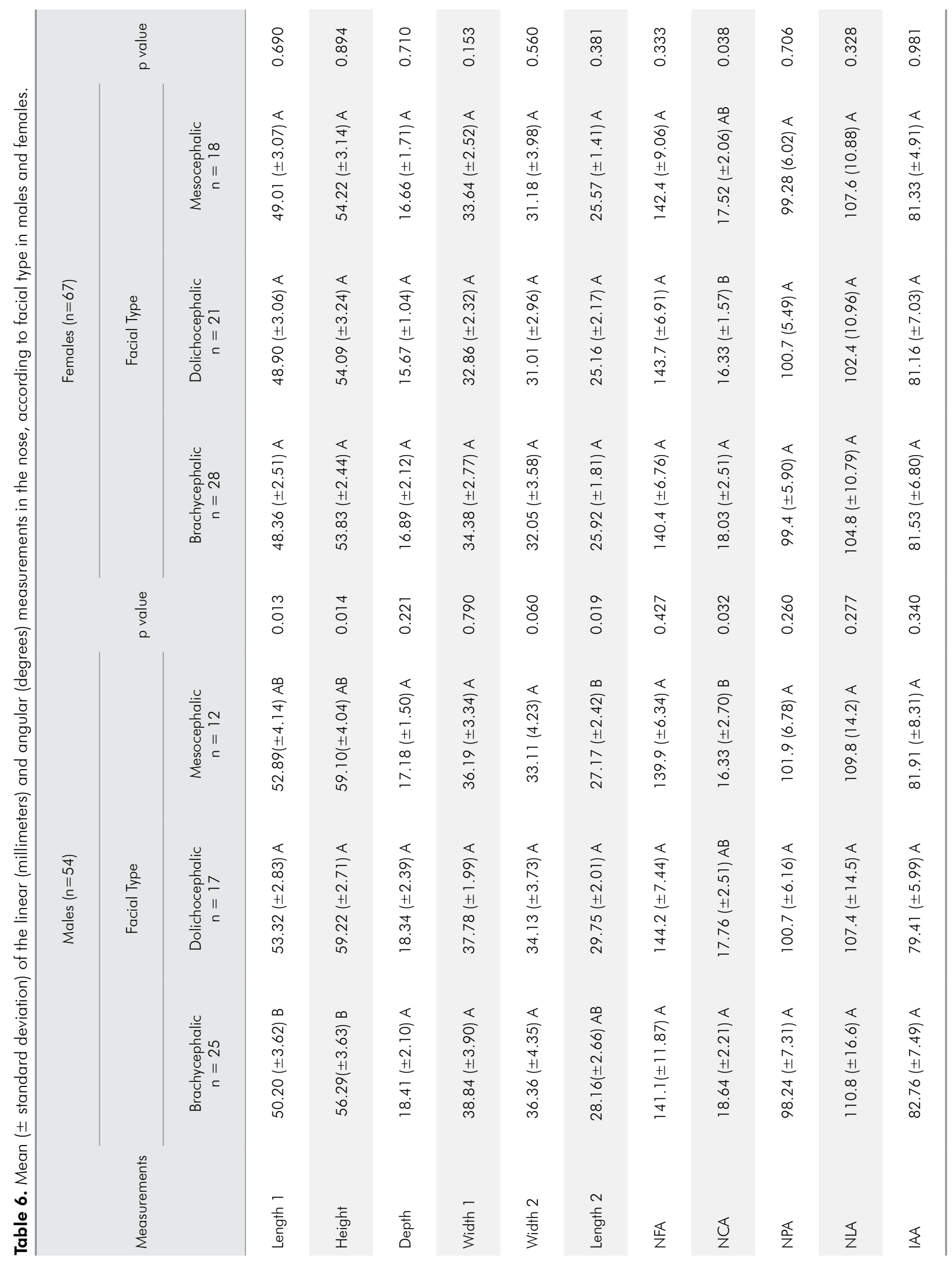


on the other hand, commonly have a vertically longer and protruding nose as well as bigger nasal bridge and root, which can be related to the greater values of length 1 , length 2 , and height found for these subjects in our study. As our study seems to be the first one on this topic, it is not possible to compare our results to the current literature. Therefore, we encourage the development of further studies in order to confirm or refute such hypotheses.

All analyses performed in the present study were carried out using CBCT images, since their use for forensic purposes, including FR, is increasing. ${ }^{15}$ The definition of soft tissues in CBCT images is considered sufficient to determine soft tissue limits, including the lateral profile of the patient. ${ }^{16}$ Additionally, high intra- and inter-examiner agreements in the assessment of soft tissue thickness in CBCT scans were found, ${ }^{29}$ suggesting that this technology is reliable for FR. Likewise, ICC results showed excellent intra-examiner agreement for all nose dimensions, thus demonstrating the reproducibility of the applied methodology. Regarding age, we only included CBCT exams of patients aged 21 years or older, as at that age, skeletal and integumentary tissues are completely developed. Additionally, as the CBCT images were acquired previously to this study and for orthodontic or orthognathic surgery planning, the oldest patient in the sample was 40 years old, since these treatments are mostly performed in relatively young patients.

Due to the variability of craniofacial characteristics within different populations, our results may not be directly applied to other ethnic groups. Additionally, as it is the first study to evaluate nose dimensions on different skeletal classes and facial types, we encourage the employment and testing of this methodology in different ethnical samples and age groups.

In summary, we evaluated the nose linear and angular dimensions of subjects of different sexes, skeletal classes, and facial types by means of CBCT images, and found that all studied factors have some influence on nose morphology. Significantly greater values were found for males in all linear dimensions of the nose, revealing sexual dimorphism. A similarity in some measurements was observed between class I and class II individuals, differing from class III subjects. Nose dimensions also differed between facial types. Therefore, we recommend that such factors be considered when performing nose reconstructions, using specific dimensions for sex, skeletal class, and facial type in regions that significantly differed and using mean values of soft tissue for regions that did not differ, in order to acquire more reliable facial approximations.

\section{Conclusion}

Nose dimensions differ in specific points among individuals of different sexes, skeletal classes, and facial types. This information may be applicable for nose reconstructions, allowing more reliable forensic facial approximations.

\section{Acknowledgements}

This research was financed in part by the Coordenação de Aperfeiçoamento de Pessoal de Nível Superior (CAPES) - Finance Code 001.

\section{References}

1. Sarilita E, Rynn C, Mossey PA, Black S, Oscandar F. Nose profile morphology and accuracy study of nose profile estimation method in Scottish subadult and Indonesian adult populations. Int J Legal Med. 2018 May;132(3):923-31. https://doi.org/10.1007/s00414-017-1758-4

2. Gomes AF, Gamba TO, Yamasaki MC, Groppo FC, Haiter Neto F, Possobon RF. Development and validation of a formula based on maxillary sinus measurements as a tool for sex estimation: a cone beam computed tomography study. Int J Legal Med. 2019 Jul;133(4):1241-9. https://doi.org/10.1007/s00414-018-1869-6

3. Rynn C, Wilkinson CM. Appraisal of traditional and recently proposed relationships between the hard and soft dimensions of the nose in profile. Am J Phys Anthropol. 2006 Jul;130(3):364-73. https://doi.org/10.1002/ajpa.20337

4. Angelakopoulos N, Franco A, Willems G, Fieuws S, Thevissen P. Clinically detectable dental identifiers observed in intra-oral photographs and extra-oral radiographs, validated for human identification purposes. J Forensic Sci. 2017 Jul;62(4):900-6. https://doi.org/10.1111/1556-4029.13310 
5. Pithon MM, Ribeiro DLR, Santos RL, Santana CL, Cruz JPP. Soft tissue thickness in young north eastern Brazilian individuals with different skeletal classes. J Forensic Leg Med. 2014 Feb;22:115-20. https://doi.org/10.1016/i.jflm.2013.09.014

6. Fernandes CM, Serra MC, Silva JV, Noritomi PY, Pereira FD, Melani RF. Tests of one Brazilian facial reconstruction method using three soft tissue depth sets and familiar assessors. Forensic Sci Int. 2012 Jan;214(1-3):211.e1-7. https://doi.org/10.1016/j.forsciint.2011.08.017

7. Utsuno H, Kageyama T, Uchida K, Yoshino M, Miyazawa H, Inove K. Facial soft tissue thickness in Japanese children. Forensic Sci Int. 2010 Jun;199(1-3):109.el-6. https://doi.org/10.1016/i.forsciint.2010.02.016

8. Thiemann N, Keil V, Roy U. In vivo facial soft tissue depths of a modern adult population from Germany. Int J Legal Med. 2017 Sep;131(5):1455-88. https://doi.org/10.1007/s00414-017-1581-y

9. Allam E, Mpofu P, Ghoneima A, Tuceryan M, Kula K. The relationship between hard tissue and soft tissue dimensions of the nose in children: a 3D cone beam computed tomography study. J Forensic Sci. 2018 Nov;63(6):1652-60. https://doi.org/10.1111/1556-4029.13801

10. Ridel AF, Demeter F, Liebenberg J, L'Abbé EN, Vandermeulen D, Oettlé AC. Skeletal dimensions as predictors for the shape of the nose in a South African sample: A cone-beam computed tomography (CBCT) study. Forensic Sci Int. 2018 Aug;289:18-26. https://doi.org/10.1016/i.forsciint.2018.05.011

11. Rynn C, Wilkinson CM, Peters HL. Prediction of nasal morphology from the skull. Forensic Sci Med Pathol. 2010 Mar;6(1):20-34. https://doi.org/10.1007/s12024-009-9124-6

12. He ZJ, Jian XC, Wu XS, Gao X, Zhou SH, Zhong XH. Anthropometric measurement and analysis of the external nasal soft tissue in 119 young Han Chinese adults. J Craniofac Surg. 2009 Sep;20(5):1347-51. https://doi.org/10.1097/SCS.0b013e3181ae41cf

13. Tedeschi-Oliveira SV, Beaini TL, Melani RF. Forensic facial reconstruction: nasal projection in Brazilian adults. Forensic Sci Int. 2016 Sep;266:123-9. https://doi.org/10.1016/j.forsciint.2016.05.004

14. Utsuno H, Kageyama T, Uchida K, Kibayashi K. Facial soft tissue thickness differences among three skeletal classes in Japanese population. Forensic Sci Int. 2014 Mar;236:175-80. https://doi.org/10.1016/j.forsciint.2013.12.040

15. Lee KM, Lee WJ, Cho JH, Hwang HS. Three-dimensional prediction of the nose for facial reconstruction using cone-beam computed tomography. Forensic Sci Int. 2014 Mar;236:194.e1-5. https://doi.org/10.1016/i.forsciint.2013.12.035

16. Scarfe WC, Farman AG. What is cone-beam CT and how does it work? Dent Clin North Am. 2008 Oct;52(4):707-30. https://doi.org/10.1016/i.cden.2008.05.005

17. Stephan CN, Simpson EK. Facial soft tissue depths in craniofacial identification (part I): an analytical review of the published adult data. J Forensic Sci. 2008 Nov;53(6):1257-72. https://doi.org/10.1111/j.1556-4029.2008.00852.x

18. Chen F, Chen Y, Yu Y, Qiang Y, Liu M, Fulton D, et al. Age and sex related measurement of craniofacial soft tissue thickness and nasal profile in the Chinese population. Forensic Sci Int. 2011 Oct;212(1-3):272.e1-6. https://doi.org/10.1016/j.forsciint.2011.05.027

19. Troncoso Pazos JA, Suazo Galdames IC, Cantín López M, Zavando Matamata DA. Sexual dimorphism in the nose morphotype in adult chilean. Int J Morphol. 2008;26(3):537-42. https://doi.org/10.4067/S0717-95022008000300005

20. Gulsen A, Okay C, Aslan BI, Uner O, Yavuzer R. The relationship between craniofacial structures and the nose in Anatolian Turkish adults: a cephalometric evaluation. Am J Orthod Dentofacial Orthop. 2006 Aug;130(2):131.e15-25. https://doi.org/10.1016/i.ajodo.2006.01.020

21. Hamid S, Abuaffan AH. Facial soft tissue thickness in a sample of Sudanese adults with different occlusions. Forensic Sci Int. 2016 Sep;266:209-14. https://doi.org/10.1016/i.forsciint.2016.05.018

22. Wang J, Zhao X, Mi C, Raza I. The study on facial soft tissue thickness using Han population in Xinjiang. Forensic Sci Int. 2016 Sep;266:585.el-5. https://doi.org/10.1016/j.forsciint.2016.04.032

23. Steiner CC. Cephalometrics for you and me. Am J Orthod. 1953 Oct;39(10):729-55. https://doi.org/10.1016/0002-9416(53)90082-7

24. Jarabak JR, Fizzell JA. Technique and treatment with light-wire edgewise appliances. 2nd ed. Saint Louis: Mosby; 1972.

25. Ricketts RM. A foundation for cephalometric communication. Am J Orthod. 1960 May;46(5):330-57. https://doi.org/10.1016/0002-9416(60)90047-6

26. Cicchetti DV. Guidelines, criteria, and rules of thumb for evaluating normed and standardized assessment instruments in psychology. Psychol Assess. 1994;6(4):284-90. https://doi.org/10.1037/1040-3590.6.4.284

27. Sforza C, Grandi G, De Menezes M, Tartaglia GM, Ferrario VF. Age- and sex-related changes in the normal human external nose. Forensic Sci Int. 2011 Jan;204(1-3):205.el-9. https://doi.org/10.1016/i.forsciint.2010.07.027

28. Umale VV, Singh K, Azam A, Bhardwaj M, Kulshrestha R. Evaluation of nasal proportions in adults with class I and class II skeletal patterns: a cephalometric study. J Orthod Sci. 2017 Apr-Jun;6(2):41-6. https://doi.org/10.4103/2278-0203.205453

29. Hwang HS, Kim K, Moon DN, Kim JH, Wilkinson C. Reproducibility of facial soft tissue thicknesses for craniofacial reconstruction using cone-beam CT images. J Forensic Sci. 2012 Mar;57(2):443-8. https://doi.org/10.1111/j.1556-4029.2011.02007.x 